\title{
Estrategias de aprendizaje y desarrollo de la motivación: un estudio empírico con estudiantes de E/LE brasileños
}

\author{
Paulo Roberto Marins de Andrade \\ Centro Universitario de Brasilia - UniCEUB \\ Becario del MAEC - AECID
}

Recibido: 16 de junio de 2009 / Aceptado: 28 de enero de 2010

ISSN: 1697-7467

\begin{abstract}
RESUMEN: Este artículo se fundamenta en la relación de algunas «estrategias de aprendizaje» propuestas por Oxford sobre la motivación. Es decir, he indagado qué actividades son más motivadoras en el estudio del E/LE según estudiantes brasileños en un contexto de aprendizaje de no inmersión. En el estudio empírico las preguntas de los cuestionarios y de la entrevista se basaron en los modelos de estrategia de aprendizaje propuestos por Oxford. Los resultados apuntan al uso de las artes como las estrategias de aprendizaje más motivadoras.

Palabras clave: auto-motivación, estrategias de aprendizaje, autonomía, afectividad, competencia de aprendizaje/existencial

«Learning strategies and motivational development: An empirical study with Spanish as a Foreign Language students in Brazil»

ABSTRACT: This article is based on the relation of some «learning strategies» especially those of Oxford about Motivation. That is, what activities are more motivational in the study of the Spanish as Foreign Language of Brazilian students in a context of learning of no immersion? In the empirical study, the questions of the questionnaires and the interviews were based on models of learning strategies proposed by Oxford. The results point to use the arts as the more motivational learning strategies.
\end{abstract}

Keywords: self-motivation, learning strategies, affectivity, autonomy, learning/existential competence

\section{INTRODUCCIÓN}

El estudio de las estrategias de aprendizaje (EA) tiene como objetivo fomentar el potencial de autonomía del estudiante y que este aprenda a regular mejor y a agilizar su proceso de aprendizaje de lengua extranjera teniendo como resultado el fomento de su competencia de aprendizaje y el aumento de los niveles motivacionales. Es sabido que la relación entre las EA y la motivación no es objeto de investigación sistemática, motivo este que nos ha llevado a investigar dicha relación. Siendo la tipología de EA extensa y no uniformada, tan sólo vamos a fijarnos en algunas de ellas sobretodo en el modelo estratégico de Oxford (1990) que las 
divide en dos grandes grupos: estrategias directas e indirectas. También vamos a contemplar, aunque sucintamente, algunos supuestos teóricos sobre la motivación, las diferencias individuales y estilos cognitivos en el aprendizaje de lenguas extranjeras.

Hemos observado en la revisión bibliográfica que los modelos de taxonomías de estrategias no están completos ni contemplan factores como la automotivación o el fomento de estrategias particulares. Entre las estrategias preferidas apuntadas por los informantes están las relacionadas con la música, el cine y la literatura, además de otras que veremos enseguida. Los objetivos y las preguntas de la investigación se han desglosado teniendo en cuenta dos ejes: el de los estudiantes y el de la profesora. Para tal fin, se aplicaron dos instrumentos de investigación, además de la observación de las clases.

Tras una revisión teórica de la literatura existente sobre el componente estratégico, el componente afectivo de la motivación y las diferencias individuales/cognitivas, procederemos al desarrollo del estudio empírico. En el estudio empírico seleccionamos una muestra de estudiantes de Español/Lengua Extranjera (E/LE) en un contexto de no-inmersión para comprobar variables como la motivación y la autonomía en el estudio de la lengua (estrategias y recursos utilizados).

Una vez elegida la muestra analizamos variables como las de contexto, presagio y proceso intentando contrastarlas con las teorías revisadas en el marco teórico para sólo entonces llegar a los resultados obtenidos con la investigación (variables de producto) en las conclusiones.

\section{MARCo teÓRICO}

\subsection{El estudio de las estrategias de aprendizaje}

Gargallo (1999:38) define el concepto de estrategias de aprendizaje en los siguientes términos: "conjunto de planes, mecanismos u operaciones mentales que el individuo que aprende una lengua pone en marcha de forma consciente para que el proceso de aprendizaje se efectúe y se agilice». Por otro lado, D. Madrid en su artículo Learning Strategies (2000) enumera algunas definiciones de EA señaladas por diversos autores que tratan este tema, entre ellas destacamos la definición aportada por Oxford (1990) ya que posee una visión más amplia del proceso: "Learning strategies are specific actions taken by the learner to make learning easier, faster, more enjoyable, more self-directed, more effective, and more transferable to new situation». Es decir, mientras otros investigadores sólo consideran el ámbito de lo cognitivo, Oxford incorpora la esfera del placer: el hecho de tornar agradable el aprendizaje.

Gargallo (1999:38) añade que esta definición tiene que ver con conceptos como la adquisición de la «autonomía en el aprendizaje» y el «autoaprendizaje». Para Küster (2004:3738) el concepto de «autonomía» está relacionado intrínsecamente con el de estrategias, pues «la investigación sobre las estrategias de aprendizaje se sitúa dentro del marco de las teorías cognitivas, las cuales conciben el estudiante como sujeto autónomo». Más adelante afirma que a su modo de ver, las estrategias son la clave para la adquisición de la autonomía. Por esta razón, el cómo aprender a aprender es muy evidente en los enfoques más recientes, que están buscando cada vez más triangular psicología, pedagogía y enseñanza de lenguas extranjeras. 
Estos «planes, mecanismos u operaciones mentales» tienen como finalidad ayudar al alumnado en la difícil tarea de adquirir o aprender la lengua objeto, reduciendo el tiempo de estudio y dinamizándolo a la vez. Oxford (1990:8) construye el concepto de estrategias desarrolladas por los aprendices para avanzar en el desarrollo de sus habilidades lingüísticas. Queda comprobar cuáles serían las más apropiadas para el aprendizaje del español/LE en particular.

De otra parte, Gargallo (op. cit.) se plantea, entre otras cuestiones, qué estrategias facilitarían el aprendizaje específicamente del español como lengua extranjera, e igualmente deja claro que hay «estrategias universales», es decir, que sirven para el aprendizaje de cualquier cosa y otras que se aplican más bien al aprendizaje de lenguas, como son las estrategias comunicativas $^{1}$. Afirma además que las diferencias individuales como la personalidad, el bagaje cultural, el origen o el estilo de aprendizaje influyen también en la elección de una u otra estrategia.

Pese a que en los últimos años ha aumentado considerablemente la teorización sobre el campo de las EA en este ámbito, los conceptos muchas veces no son convergentes y tampoco su clasificación como puede comprobarse en las diferentes taxonomías que se han llevado a cabo. Según Díez et al. (2004:10) hay una carencia de una base empírica clara a la hora de establecer las taxonomías, pues, si muchas de estas se basan en la intuición o en la observación ad hoc de sus autores no se llegará a un consenso en cuanto a estas, ni tampoco acerca de cómo sostener una investigación o práctica docente fundamentada. Valcárcel, Coyle y Verdú (1996:87-88) a este respecto nos comentan:

«The problem, however, is that in the available research literature there is no consensus of opinion as to the number or indeed the names attributed to these learning processes and strategies. As Ellis (1985:166) points out 'the metalanguage involved in the cognitive components of procedural knowledge is often confusing and vague'. Researchers do not use terms like process and strategy consistently. Sometimes they are used as synonyms for general mental operations and at other times to differentiate operations involved in language processing (...) LA strategies researchers will continue to offer vying and biases taxonomies of language learning strategies claiming 'my set of categories is better than yours' (Cook 1993)» .

No existe, tampoco, un consenso si las EA son procesos conscientes, automatizados o inconscientes. Sin embargo, Küster (2004:38) considera que parece existir un consenso en que las EA son «accesibles a la consciencia individual». Por otro lado, Fernández López (1996:5) según el grado de mayor o menor conciencia o intencionalidad divide las estrategias en dos grupos:

Sin entrar en la disquisición de las diferentes propuestas, creemos que se puede trazar una línea con dos polos, de menor a mayor consciencia o intencionalidad, para diferenciar aquellos mecanismos, operaciones mentales que rigen los procesos humanos del aprendizaje, y que habitualmente actúan de forma inconsciente, de los

${ }^{1}$ En cuanto a la estructura interna de las estrategias, el esquema más amplio es una vez más el de Oxford -pero tan extenso que no siempre permite diferenciar estrategias de aprendizaje de estrategias de comunicación-. (Küster, 2004:38). 
recursos, técnicas, procedimientos, trucos, que si no son intencionados, sí podemos reconocer fácilmente en una introspección sobre nuestra forma de aprender.

A este respecto Franco Naranjo (2004:64) también está de acuerdo de que «La mayoría de los alumnos, en la práctica de las destrezas, son más concientes de las estrategias cognitivas y metacognitivas que intervienen en el procesamiento de la información y en la organización y evaluación del aprendizaje, que de los aspectos afectivos y sociales».

Para reconocer el papel de las estrategias en el aprendizaje de idiomas y su importancia es menester tener una visión ampliada de los porqués y finalidades del aprendizaje de una lengua. Por tanto, el fin último del aprendizaje de idiomas es la adquisición de la competencia lingüístico-comunicativa y tal hecho se logra por medio de la elección adecuada de actividades por parte del/de la docente y por el uso apropiado de las EA por parte del/de la discente.

Así, cuando el/la aprendiz consciente o inconscientemente elige estrategias que se ajustan a su estilo de aprendizaje, estas se tornan sus «herramientas útiles» para activar y regular todo el proceso y obtener resultados estables. Consecuentemente, el profesorado debe tener el compromiso de ayudar a su alumnado seleccionando las estrategias más adecuadas a sus características con vistas a optimizar resultados para hacerlos significativos y de calidad (Alonso Tapia y Cartula Fita, 2003:98). Fonseca Mora (op. cit:109) también lo avala al afirmar que los aprendices de secundaria tienen excelentes destrezas para superar muchos problemas de aprendizaje, no obstante, necesitan aprender cómo hacerlo y entender el porqué de hacerlo.

Podemos concluir que es preciso que el docente no sólo sepa adecuar las estrategias a los estilos de aprendizaje sino que también pueda elegir apropiadamente la metodología y las actividades intentando conjugar materiales, manual, diferencias y creencias personales con vistas al desarrollo global del estudiante.

\subsection{El Modelo Estratégico de Oxford}

Oxford (1990) es quien ha proporcionado una de las clasificaciones de EA que goza de mayor acogida por parte de los expertos internacionales en lingüística aplicada. No obstante, O’Malley y Chamot (1985) han elaborado una clasificación más completa, además de Valcárcel, Coyle y Verdú (op.cit.) que ofrecen una clasificación más detallada, según datos de McLaren y Madrid (1996). Además, Oxford (op. cit.) intenta armonizar emociones, cognición y placer por lo que ha hecho una importante contribución en este campo. La tipología de EA establecida según Oxford (1990 apud Gargallo 1999: 41) además refleja el conjunto de planes que el hablante no nativo pone en marcha para contribuir al aprendizaje y poder lograr la competencia comunicativa.

La autora divide las EA en seis subdivididas en dos grandes grupos: Estrategias Directas e Indirectas, como podemos observar en el siguiente cuadro: 
Cuadro 1: Estrategias de aprendizaje según Oxford (Adaptado de Gargallo, 1999:41)

\begin{tabular}{|c|c|}
\hline \multicolumn{2}{|c|}{ ESTRATEGIAS DE APRENDIZAJE } \\
\hline Estrategias Directas & Estrategias Indirectas \\
\hline $\begin{array}{c}\text { DE MEMORIA } \\
\text { (Para retener y acordarse de las nuevas } \\
\text { informaciones) }\end{array}$ & $\begin{array}{c}\text { METACOGNITIVAS } \\
\text { (Para coordinar el proceso de aprendizaje) }\end{array}$ \\
\hline $\begin{array}{l}\text { 1. Crear asociaciones mentales } \\
\text { 2. Asociar imágenes y sonidos } \\
\text { 3. Dar respuestas físicas }\end{array}$ & $\begin{array}{l}\text { 1. Delimitar lo que se va a aprender } \\
\text { 2. Ordenar y planear lo que se va a } \\
\text { aprender } \\
\text { 3. Evaluar el aprendizaje, analizando los } \\
\text { problemas y buscando soluciones }\end{array}$ \\
\hline $\begin{array}{c}\text { COGNITIVAS } \\
\text { (Para dar sentido al aprendizaje y producir } \\
\text { el lenguaje) }\end{array}$ & $\begin{array}{c}\text { AFECTIVAS } \\
\text { (Para regular las emociones) }\end{array}$ \\
\hline $\begin{array}{l}\text { 1. Practicar los contenidos comunicativos } \\
\text { 2. Codificar y descodificar mensajes } \\
\text { 3. Analizar y razonar } \\
\text { 4. Utilizar recursos para organizar la } \\
\text { información y poder utilizarla } \\
\end{array}$ & $\begin{array}{l}\text { 1. Reducir la ansiedad } \\
\text { 2. Animarse } \\
\text { 3. Controlar las emociones }\end{array}$ \\
\hline $\begin{array}{c}\text { COMPENSATORIAS } \\
\text { (Para ayudar a los estudiantes a vencer lagunas } \\
\text { de conocimientos para continuar la } \\
\text { comunicación) }\end{array}$ & $\begin{array}{c}\text { SOCIALES } \\
\text { (Para que el estudiante aprenda con los } \\
\text { demás aumentando su nivel de } \\
\text { interacción con la lengua objeto) }\end{array}$ \\
\hline $\begin{array}{l}\text { 1. Adivinar el sentido } \\
\text { 2. Resolver problemas de comunicación } \\
\text { (Estrategias de comunicación) }\end{array}$ & $\begin{array}{l}\text { 1. Pedir aclaraciones, verificaciones o } \\
\text { repeticiones } \\
\text { 2. Interactuar con hablantes nativos y } \\
\text { hablante no nativos. } \\
\text { 3. Empatizar con los demás }\end{array}$ \\
\hline
\end{tabular}

Oxford (1990:14-16) hace una analogía entre las estrategias directas e indirectas con el teatro. La primera gran clase de estrategias, -las directas - se asemejan a un artista en el palco, que trabaja con el lenguaje en sí y una variedad de nuevas situaciones y tareas específicas. El artista usaría este grupo de estrategias muy próximo al director, como pendiente de su opinión para una actuación mejor. El grupo de las indirectas se compara al papel que ejerce el director de la pieza (quien en realidad las prepara para el proceso de aprendizaje). Podemos así concluir que las estrategias indirectas fomentarían aún más la autonomía del estudiante que las directas, pues tanto las funciones del director como las del artista son responsabilidad del alumno.

A pesar de la amplitud de la tipología de EA brindadas por Oxford sugeriríamos que se ampliaran las de corte afectivo y de (auto)motivación, por lo que, en este sentido, estamos de acuerdo con Küster (op. cit.).

También merecen ser destacados otros autores españoles e internacionales en la clasificación y estudio de las estrategias como: D. Madrid (2000), Sonsoles Fernández (1996, 2005), 
Valcárcel, Coyle y Verdú (1996), Fonseca Mora (2005), Manchón, R. y Bruton, A. (2005), además de Ellis (1985), los mismos O'Malley y Chamot $(1985,1989,1990)$ entre otros. También merecen destaque los artículos de Sagnier (2005), Franco Naranjo (2004), Fernández Toledo (2006), Morales y Smith (2008) y Marefat y Barbari (2009). No obstante, la mayoría de los investigadores sólo contempla el ámbito de lo cognitivo. O’Malley y Chamot (op. cit.) distinguen no sólo estrategias cognitivas o metacognitivas sino también las socioafectivas.

Para concluir, según las características generales de las EA que Oxford (1990) destaca, éstas contribuyen sobre todo a fomentar la autonomía de los estudiantes, lo que no significa que acorten el trabajo del profesorado. Por el contrario, ellas favorecen la labor del docente ampliando su rol de mediador y motivador en el aprovechamiento de estas por el alumnado. Además, son influenciadas por las diferencias individuales, los estilos cognitivos y la motivación.

\subsection{La motivación: armonización entre emociones y cognición}

La motivación es el componente afectivo más estudiado por los investigadores de lenguas extranjeras y el que guarda mayor relación con el uso de las estrategias. Además, es sabido que es el componente afectivo que congrega la conjugación perfecta entre emociones y cognición, es decir, «puede ser representada a partir de un estado de activación cognitiva y emocional» (Williams y Burden, 1999:128). En este sentido, el concepto de motivación se relaciona estrechamente con el de las estrategias de Oxford que también intenta compaginar lo cognitivo con lo emocional. Dicho concepto conlleva una decisión consciente de actuar y da lugar a un período de esfuerzo intelectual y/o físico sostenido con el fin de lograr una meta o metas previamente establecidas. Así, el uso de EA acarrea el fomento de la motivación y viceversa: es decir, si el estudiante está motivado tratará de desarrollar estrategias; si ya las posee, las utiliza y si le funcionan crece aún más su motivación.

Martínez Lirola (2005:26) también afirma que la motivación es uno de los aspectos psicológicos más importantes en el proceso educativo pues los que están más motivados aprenden más y con más eficacia:

«la motivación o deseo de aprender es un factor fundamental en el aprendizaje; podemos considerarla como uno de los aspectos más importantes en el proceso educativo. Se trata de un factor psicológico que pone de manifiesto el éxito de los estudiantes a la hora de aprender una lengua extranjera. Nos atrevemos a afirmar que los alumnos con un alto grado de motivación aprenden antes y obtienen mejores resultados en el aprendizaje de una lengua extranjera» (...)

La motivación puede depender de factores externos (influencia de otras personas o algún suceso), o internos (interés o curiosidad) de modo que podemos clasificarla en intrínseca o extrínseca; en integradora o instrumental ${ }^{2}$. Arnold y Brown (2000) defienden la postura de

2 La motivación extrínseca nace del deseo de conseguir una recompensa: también puede nacer del deseo de evitar alguna punición. Así, su foco está en algo externo a la actividad de aprendizaje. La motivación intrínseca supone que la experiencia de aprendizaje es su propia recompensa: «la curiosidad y el interés naturales de los alumnos potencian el aprendizaje (Deci y Ryan 1985: 245). «La motivación extrínseca nace del deseo de conseguir una recompensa (...) Así su foco está en algo externo a la actividad de aprendizaje» 
que el desarrollo de la motivación intrínseca revierte en un mayor éxito por parte del alumnado fomentándose así tanto su potencial de autonomía como la competencia de aprendizaje. También se ha probado que la motivación externa (el feedback, elogios) acrece igualmente la motivación intrínseca (sentido personal que cada quien delega a los estímulos externos). Consideramos que la motivación extrínseca también puede funcionar como una eficaz «estrategia de control afectivo» cuando el alumnado las utiliza conscientemente. Gardner (2007:19), por su parte, afirma que las distinciones de clases de motivación no son lo verdaderamente relevante sino su calidad e intensidad en sentido amplio, al incorporar las actitudes, los factores cognitivos y, desde luego, el componente afectivo. Por ejemplo, Morales y Smith (2008: 177) demuestran que la estrategia del uso de imágenes mentales y visuales aliada al factor motivacional constituye un importante elemento de aprendizaje afectivo:

Así, son muchos los autores que tratan del tema de la motivación en el aprendizaje de idiomas. Merecen destaque los artículos de R. C. Gardner (2007) «Motivation and second Language Adquisition»; Saravia y Bernaus (2008) «Motivación y actitudes para el Aprendizaje de Lenguas de Dos Colectivos de Estudiantes Universitarios(...)»; Bernaus, Wilson y Gardner (2009) «Teachers motivation, classroom strategies use, students' motivation and second language achievement» y el artículo de Padial Campón y Tapia Carrillo (2007) titulado «The Motivation to use oral language in the ESL classroom in Secondary Education» trata sobre la relación entre la inhibición del uso oral de la lengua y la motivación.

Consideramos que para ampliar el concepto de «competencia de aprendizaje» o el potencial de autonomía del estudiante habría que incrementar la «automotivación», para que el/la alumno/a fuera capaz de buscar sus propias fuentes motivacionales lo que llamaríamos «competencia motivacional», que podría adquirirse mediante el uso de las EA y por el descubrimiento de otras de carácter personal según sus estilos cognitivos, personalidad, diferencias, cultura y valores, por ejemplo.

\subsection{La importancia de las diferencias individuales en el estudio de las estrategias}

Partiendo de la premisa de que el aprendiz es «el eje de todo proceso didáctico» con sus motivaciones, actitudes, aptitudes, inteligencia, cultura, idiosincrasia, capacidades, etc., hace falta destacar algunos aspectos relacionados con los «estilos y diferencias de aprendizaje». Hay toda una línea de investigación que intenta describir estos aspectos relacionándolos con lo anteriormente dicho, así como las consecuencias de estas diferencias en los estilos de aprendizaje y en la acción didáctica. Según Sonsoles Fernández (2005:413):

(...) toda una línea de investigación (Skehan, 1989; Oxford, 1989; Naiman y otros, 1978) se preocupa con estudiar las diferencias individuales de los aprendices -edad, necesidades, cultura, lengua materna, aptitud, inteligencia, actitud, personalidad $-\mathrm{y}$

\footnotetext{
como el hecho de intentar el/la estudiante satisfacer más a los docentes, a los centros escolares o a las autoridades que a sí mismo/a. (Arnold y Brown, 2000:31) Gardner y Lambert (1972) apud Arnold y Brown (2000:30) vuelven a dividir la motivación en dos más orientaciones más: integradora e instrumental. La motivación integradora es generada intrínsecamente por percepciones positivas hacia el aprendizaje de la lengua extranjera. La motivación instrumental es inducida por la influencia de fuerzas externas como, por ejemplo, el hecho de superar un examen de posición (según Martínez Agudo, 2001).
} 
las consecuencias de esas diferencias en los estilos de aprendizaje (Willing, 1988) y en los resultados obtenidos. La conclusión más clara de estos trabajos, no es que tengan más éxito los niños o los adultos, los hispanos o los chinos, los extrovertidos o los introvertidos, los auditivos o los visuales, etc. sino que las estrategias que se utilizan para aprender son muchas veces diferentes y que en todos los casos se aprende mejor cuando se aplican las estrategias apropiadas.

En cuanto a los estilos de aprendizaje, estos no son dicotómicos pues una persona puede ser más extrovertida que introvertida o igualmente visual o auditiva, así como puede aprender mejor individualmente y, sin embargo, disfrutar también con trabajos grupales. Unos estudiantes aprenden mejor con muestras orales de lengua y otros, en cambio, con muestras escritas; además, hay quienes necesitan manipular objetos y/o desarrollar una actividad física. Así, según la percepción sensorial los estilos pueden ser agrupados en cuatro áreas principales relativas a la percepción: visual, auditiva, cinestésica y táctil, además de las áreas de los sociológicos: grupal e individual. Por otro lado, hay diferencias de estilos según el tipo de personalidad (extravertidos/introvertidos, intuitivos/sensitivos, reflexivos/sentimentales, estimadores/ perceptores); según las diferencias biológicas (biorritmos, sostenimiento y la ubicación) y el temperamento (sanguíneo, melancólico, flemático y colérico).

Díez (2004:11) afirma que la relación entre estrategias y estilos de aprendizaje carece de claridad. Por otro lado, es cada vez más remota la posibilidad de que los estilos y las EA no mantengan ninguna vinculación pues, según investigaciones de Ehrman et al. (2003:315), los estilos de aprendizaje se manifiestan cuando el alumno elige la EA que corresponde a su estilo, y cuando, por tanto, esta selección no se realice al azar. Además, Ehrman (idem) afirma que quizás la instrucción de estrategias no ha obtenido resultados estables precisamente porque no se han tenido en cuenta las necesidades y los estilos de aprendizaje de los estudiantes.

Según el estudio de Martínez Lirola (2005:31) con alumnos inmigrantes norteamericanos con problemas de disciplina, fueron tomadas algunas medidas sugeridas por Prodromou (1995) que consideraron muy eficaces teniendo en cuenta la edad de los alumnos, como nos afirma: «Finalmente, de las siete medidas propuestas, fue muy eficaz cambiar la actividad comunicativa a otra en la que los alumnos tengan que trabajar de manera individual, como por ejemplo, cambiar de una actividad oral a un ejercicio escrito».

Pensamos que es reconocidamente ventajoso hacer conscientes al alumnado de sus cualidades y diferencias o preferencias individuales, pues creemos que se aumentaría el nivel de motivación e interés en el proceso de aprendizaje y les imbuiría de mayor responsabilidad y autonomía. Tanto es así que si los estilos y diferencias individuales no coinciden con las estrategias elegidas se puede provocar un sentimiento de «grave ansiedad» o hasta incluso el fracaso en el aula de idiomas, según datos de Capelle, 1995 y Ehrman, 1996 (apud Arnold 2000:318). 


\section{Metodología de la investigación}

\subsection{Objetivos de la investigación}

Partiendo de la idea de que el uso de EA puede influir positivamente en la forma en que los aprendices regulen su proceso de aprendizaje aumentando sus niveles de motivación y autonomía se han planteado los siguientes objetivos de investigación:

3.1.1. Analizar qué estrategias utilizan los/las alumnos/as para trabajar de forma más autónoma, para automotivarse y para aumentar su autoestima.

3.1.2. Averiguar cómo el profesor/a de E/LE trabaja la competencia existencial en las clases de E/LE y cómo utiliza o fomenta las estrategias motivadoras.

De este modo se puede contemplar el problema bajo dos perspectivas diferentes para compararlas: la del alumnado y la del profesorado.

\subsection{Instrumentos}

Según Sonsoles Fernández (2005) para reconocer las EA de la lengua extranjera, para saber cuáles son las que aporta el aprendiz, las más rentables, las propias de cada cultura, de cada estadio de aprendizaje y cómo se desarrollan, hace falta seguir algunos procedimientos. Así, la autora (idem) sugiere las siguientes técnicas: «análisis de las producciones de los aprendices, búsqueda de la causa de los errores, introspección y verbalización de los procesos internos, observación (guiada) de las estrategias de otras personas, entrevistas retrospectivas, cuestionarios y diarios» (p. 415-6). Empíricamente, Rubin (1981 apud O’Malley et al. 1985:26) identificó EA por medio de una variedad de otros procedimientos como grabación de clases, observaciones de situaciones de tutorías, narrativas, historietas, relatos, diarios, etc. Por todo ello, nos hemos decidido aplicar tres instrumentos de investigación: cuestionarios, entrevistas retrospectivas (verbalización de los procesos internos) y observación de clases conforme describiremos a continuación.

\subsubsection{Cuestionarios}

En el cuestionario aplicado a los estudiantes se buscó inquirir sobre la relación de las EA y el aumento de los niveles de motivación, autonomía y autoestima. Algunas de las estrategias descritas por los estudiantes son personales y no coinciden con la tipología de EA investigadas anteriormente. También se elaboró un cuestionario para el profesorado del centro con preguntas similares; sin embargo, para este trabajo analizamos sólo el de la profesora del grupo investigado.

El cuestionario se inspiró en las estrategias indirectas de Oxford, pero también se basó en otros modelos de cuestionarios como los de Giovannini et al. (2000). Estas son las preguntas hechas del cuestionario:

\subsubsection{Entrevista}

La entrevista estructurada fue realizada al final del curso para comprobar cómo se sintieron los informantes al culminar esta etapa del proceso de enseñanza-aprendizaje. Las en- 
trevistas se grabaron en audio y fueron transcritas a posteriori. Las preguntas están estrechamente relacionadas con los propósitos de nuestra investigación, aparte de servir como soporte para aclarar algún punto vago o que nos ha suscitado interés en el cuestionario así como durante las clases analizadas.

\subsection{Variables de Contexto}

Se eligió para la presente investigación un Centro Público de Lenguas en la capital de Brasil. Las clases tienen una duración de una hora y veinte minutos y con una frecuencia de dos veces semanales. La escuela está constituida por 12 aulas equipadas con televisores y vídeos además de radiograbadores.

\subsection{Variables de Presagio}

\subsubsection{Alumnado informante}

La muestra elegida para el estudio empírico consta de diecisiete estudiantes de E/LE del nivel Intermedio (nivel B1 del Marco Común Europeo de Referencia para las Lenguas). Todos son brasileños y llevan un promedio de tres años estudiando la lengua. Se solicitó por escrito a catorce estudiantes que participaran en la presente investigación y todos firmaron aceptando. A continuación describiremos las características de los informantes cuyos datos personales fueron protegidos bajo pseudónimos elegidos por ellos mismos como se puede contemplar en el cuadro siguiente:

Cuadro 2: Alumnado informante

\begin{tabular}{|c|c|c|c|c|}
\hline PSEUDÓNIMO & EDAD & GÉNERO & PROFESIÓN & $\begin{array}{c}\text { TIEMPO QUE } \\
\text { ESTUDIA } \\
\text { ESPAÑOL } \\
\end{array}$ \\
\hline 1. ANA & 27 & Femenino & Abogada & 3 años \\
\hline 2. ANE & 23 & Femenino & Estudiante & 2 años \\
\hline 3. EDU & 21 & Masculino & Estudiante & 3 años \\
\hline 4. FURICO & 19 & Masculino & $\begin{array}{l}\text { Estudiante y } \\
\text { "trabajador } \\
\text { en prácticas" }\end{array}$ & $31 / 2$ años \\
\hline 5. GURIA & 17 & Femenino & Estudiante & 3 años \\
\hline 6. JESÚS & 21 & Masculino & $\begin{array}{l}\text { Estudiante y } \\
\text { "trabajador } \\
\text { en prácticas" }\end{array}$ & \\
\hline 7. K TATAL & 19 & Masculino & En el paro & $31 / 2$ años \\
\hline $\begin{array}{l}\text { 8.LAURA } \\
\text { FERRARI }\end{array}$ & 18 & Femenino & Estudiante & $3 \frac{1}{2}$ años \\
\hline 9. LOLA & 25 & Femenino & Psicóloga & 3 años \\
\hline 10. MACHADO & 28 & Femenino & Administradora & 3 años \\
\hline 11. MARI & 16 & Femenino & Estudiante & 3 años \\
\hline
\end{tabular}


Cuadro 2: Alumnado informante (Cont.)

\begin{tabular}{|l|c|c|c|c|}
\hline $\begin{array}{c}\text { 12.MARGI } \\
\text { SIMPSON }\end{array}$ & 17 & Femenino & Estudiante & 3 años \\
\hline 13. PILAR & 21 & Femenino & Estudiante & 3 años \\
\hline 14. JUAN & 47 & Masculino & $\begin{array}{c}\text { Profesor de la Red } \\
\text { Pública }\end{array}$ & 3 años \\
\hline 15. SIMBA & $\begin{array}{c}\text { No ha } \\
\text { contestado }\end{array}$ & Femenino & $\begin{array}{c}\text { Técnica de análisis } \\
\text { de Laboratorio }\end{array}$ & 3 años \\
\hline 16. TATA & 22 & Femenino & Estudiante & 3 años \\
\hline 17. VAMPIRA & 19 & Femenino & Estudiante & 3 años \\
\hline
\end{tabular}

\subsubsection{Profesora Informante}

La profesora del grupo investigado tiene 27 años y es de origen brasileño, por lo que su lengua materna es el portugués. En el momento de realizar la investigación, completaba cinco años en la docencia del E/LE en Brasil. Según aclara en su cuestionario, en los últimos años no ha participado en ningún curso de perfeccionamiento, taller o congreso de profesores.

\subsection{Variables de Proceso}

El centro investigado adopta una metodología dinámica y un «enfoque comunicativo» para que el alumno sea capaz de expresarse, de entender, de interpretar y de comunicarse con su interlocutor. En su Orientación Pedagógica (2002) 3 se considera que las estrategias construyen y rescatan la confianza del alumnado eliminando barreras que puedan impedir su aprendizaje. Además, la metodología puede ser adaptada a las especificaciones de cada grupo. De este modo, la propuesta pedagógica de esta institución, tiene en cuenta las EA así como las diferencias individuales.

También analizamos una serie de clases para comprobar algunas acciones observables de estos informantes que fueron grabadas en vídeo con la debida autorización por escrito a cada uno de ellos.

\section{AnÁlisis y discusión de los Resultados}

Aparte de que el/la aprendiz pueda desarrollar sus propias EA particulares («estrategias del aprendiz») el profesor puede de manera sistemática y concienzuda sugerirle otras, para que este pueda elegir las que mejor se ajusten a sus características personales y a la situaciónproblema presentada ${ }^{4}$, pues «(...) el conocimiento de estas estrategias por parte del profesor

${ }^{3}$ Documento que normaliza y uniforma todos los Centros Públicos de Lenguas del Distrito Federal, diseñado por la Secretaría de Estado de Educación -SEE/DF- (2002).

${ }^{4}$ Chamot y O’Malley, según Hill (2004:21), diferencian «estrategias del aprendiz» que serían las desarrolladas por los propios estudiantes, de «estrategias de aprendizaje» refiriéndose a las presentadas explícitamente en la clase. 
y/o investigador es fundamental para conocer el perfil estratégico del alumno y le ayudará a tomar decisiones en cuanto a su actuación en el aula, por ejemplo» (Gallardo, op. cit.).

En el estudio empírico se le preguntó al alumnado informante sobre el conocimiento y uso de EA y se llegaron a unos resultados interesantes. Claro está que el cuestionario utilizado se basó, parcialmente, en la tipología de estrategias de Oxford, pero también en otros tipos de estrategias; incluso, al entrevistarles se les pregunta sobre el uso de EA propias. A continuación vamos a exponer algunos de estos resultados del cuestionario aplicado a la muestra de alumnado en cuestión analizándolos a la luz de las teorías revisadas.

\subsection{Resultado de las preguntas del cuestionario y de la entrevista ${ }^{5}$}

La primera pregunta fue inspirada en algunas EA afectivas y sociales (Oxford) como las técnicas de relajación para reducir la ansiedad; la autorecompensa como estrategia de autoanimación y la interacción con otros compañeros.

\section{1. ¿Qué estrategias de aprendizaje utilizas?}

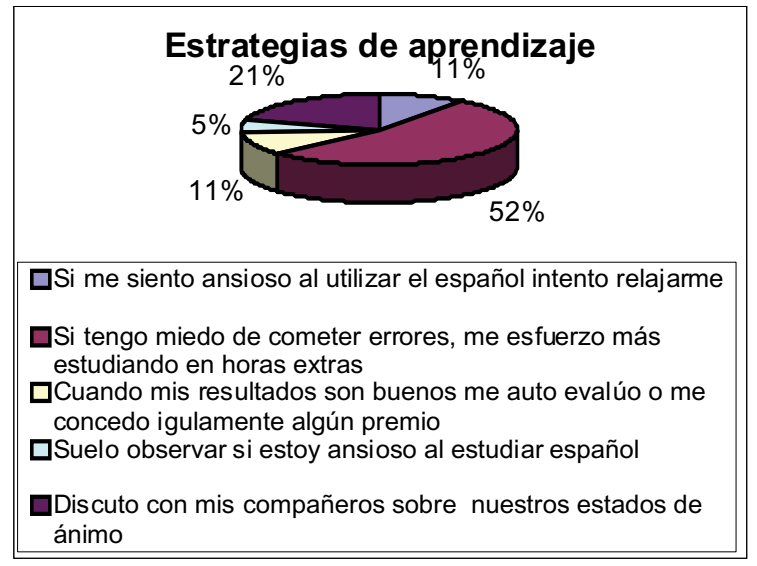

Como la mayoría ha marcado la opción «Si tengo miedo de cometer errores, me esfuerzo más estudiando en horas extras», consideramos que un cierto grado de ansiedad, por el miedo de los aprendices a equivocarse, es bueno pues les estimula a que estudien más y, por consiguiente, aumenten su grado de autonomía o locus de control. También se ha destacado el hecho de que traten con los compañeros sobre sus estados de ánimo (21\%). Estas respuestas demuestran su preocupación por la cuestión anímica y la necesidad de poner en común cuestiones afectivas tanto como estrategias para solventarlas.

La segunda pregunta tiene que ver con el uso de EA para desarrollar la autonomía:

${ }^{5}$ Evidentemente en este artículo no aparecen todas las preguntas y respuestas de los cuestionarios y entrevistas sino sólo las de mayor relieve para este estudio en particular. 


\section{2. ¿Qué estrategias utilizas para estudiar solo?}

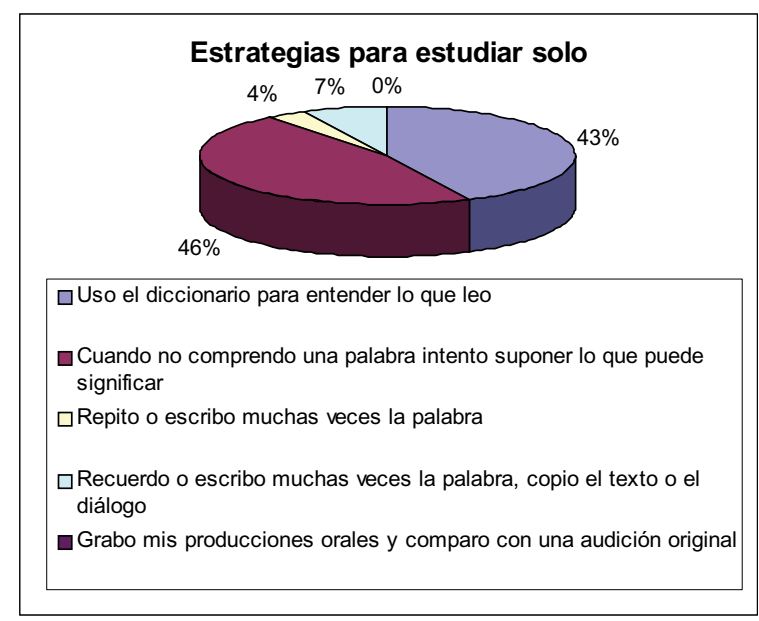

Según el modelo de Oxford, este tipo de EA descritas se clasificarían como «cognitivas»: traducir las palabras, analizar contrastivamente -comparando con la audición original (analizar y razonar) - y practicar (repetir utilizando un sistema escrito).

Entre las respuestas más frecuentes destaca la de intentar suponer lo que puede significar $(46 \%)$ partiendo del contexto o de la situación. Otra gran parte de los informantes ha confesado hacer bastante uso del diccionario para entender lo que lee $-43 \%$ - (estrategia compensatoria «directa», según Oxford). Estas respuestas demuestran el poco conocimiento y/ o uso de EA. Las estrategias inspiradas en métodos audiolinguales no fueron señaladas por nadie, como el uso de grabaciones $(0 \%)$-técnicas de laboratorios de lenguas- y en cuanto a los ejercicios de repetición, mecánicos o estructurales los señalaron el 4\% y el 7\%.

La próxima pregunta hace referencia a la relación que se da entre EA y (auto)motivación.

\section{3- ¿Qué estrategias utilizas para automotivarte en el estudio de la lengua española? Coló- calas en orden de importancia.}

- Veo películas en español

- Busco reunirme con los compañeros para estudiar en grupo

- Escucho canciones en español e intento analizar la letra

- Intento leer más literatura en español

- No sé automotivarme

- Me relaciono con nativos

A pesar de que Oxford no haya contemplado las estrategias de automotivación, nosotros las consideramos relevantes. No obstante, en las opciones que se sugieren para contestar a esta pregunta se observa una mezcla de las estrategias afectivas (escuchar música); cognitivas (ver películas en lengua extranjera -codificar y descodificar mensajes-) con las sociales (reunirse con los compañeros, relacionarse con los nativos). Por otra parte, estas respuestas no contem- 
plan otras EA personales, por lo que reformulamos la cuestión en la entrevista como veremos en la pregunta número 7.

Los resultados apuntan a que el trabajo con las canciones, la lectura y el visionado de películas en español son las estrategias de motivación del alumnado que más se destacaron. El hecho de estudiar en grupo no les funciona como estrategia de automotivación: se puede deducir que estos informantes prefieren un uso individual de las EA frente al grupal.

La siguiente pregunta atañe más bien al estilo de aprendizaje del estudiantado encuestado y a la cuestión del nivel neurológico del ambiente (ubicación), según la teoría de los estilos de aprendizaje, puesta en relación con el potencial de autonomía y el uso de estrategias.

\section{4. ¿Cómo piensas que aprendes mejor?}

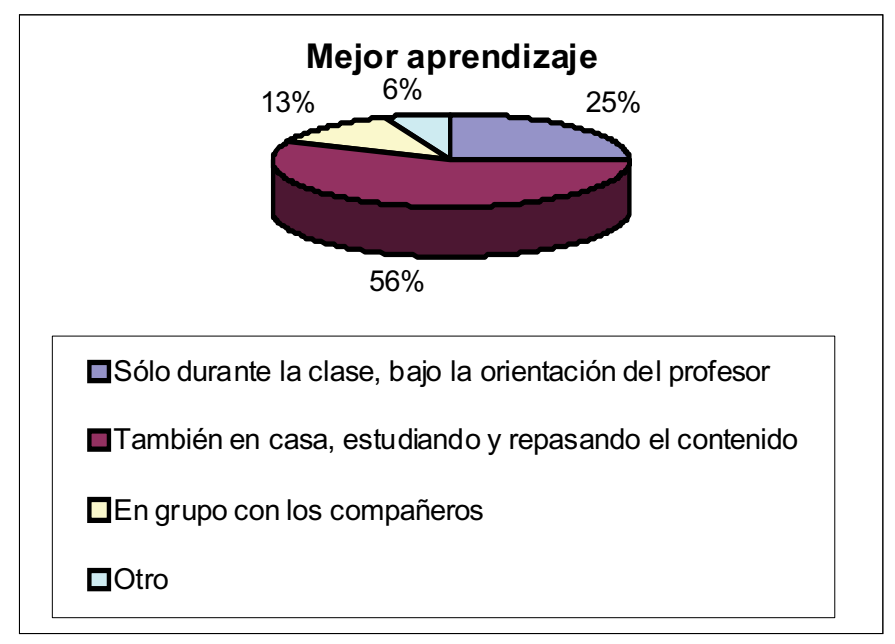

Estos tipos de EA pueden ser clasificados como una mezcla entre destrezas de memoria (revisión del contenido) y estrategias afectivas-sociales (hacer preguntas al profesor, interaccionar con los compañeros en grupos).

Así como la mayoría (el 56\%) ha marcado que aprende mejor «en casa» y «sólos» estudiando y repasando, deducimos que tienen consciencia de que su papel como estudiantes autónomos es importante y mantienen una visión individualista. Para ser autónomos prefieren estar solos y no en grupos; en otras palabras, su estilo de aprendizaje es más individual que grupal. Esta visión solitaria de la autonomía aún la hace más árida ya que la reclusión no les facilita el intercambio de estrategias y tampoco fomenta el debate, que fuera del aula puede ser tan enriquecedor como en clase. Sugerimos que el profesorado debería fomentar este tipo de prácticas grupales.

Comparamos a continuación algunas respuestas de la entrevista efectuada a los informantes: 


\section{Cuando te sientes desmotivado, ¿qué haces para automotivarte, qué estrategias utilizas?}

Agrupando a los/las informantes por respuestas parecidas obtuvimos estos resultados: Juan, Lola, Machado, Ana y Simba están de acuerdo en que las películas son, entre otras, una eficaz estrategia de automotivación. Machado igualmente destaca cualquier tipo de arte como elemento automotivador. Ana y Lola destacan también el hecho de hablar con personas que ya sean oralmente competentes en la lengua objeto y Lola, Ana, Guria, Simba y Jesús confiesan que deben leer en español, ya sean diálogos o literatura, para familiarizarse más con el idioma. Jesús reconoce que aprovecha los textos interesantes del propio libro utilizado en clase. Machado, Mari, Guria, Ane y Laura Ferrari destacan la música ${ }^{6}$ como estrategia automotivadora. Laura Ferrari y Ane intentan cantar, la primera vez tratando de «imitar la letra de la canción». Machado aprende nuevo vocabulario con la canción y Mari confiesa que la música es lo único que le hace sentirse bien.

Además de la música y el cine como recursos auto-motivadores, hubo algunos/as que presentaron sugerencias diversas, como Edu que para automotivarse intenta pensar cuándo va a emplear el español en su vida diaria, ya que considera que va a ser muy importante para él en el futuro (estrategia de motivación extrínseca). La estrategia de Tata es observar si está ansiosa al estudiar español («escucha su propio cuerpo»-estrategia afectiva-Oxford -). K Tatal reconoce que su estrategia es pensar que los españoles están hablando cerca de él y tratar de tener más calma (técnica de relajación -Oxford -). Vampira es la única que cuando se siente desmotivada no utiliza ninguna estrategia para automotivarse.

Es decir, lo que más motiva a cada uno depende de sus características: preferencias sensoriales y diferencias de personalidad. A modo de conclusión, consideramos que estas respuestas demuestran una tendencia a categorizar las artes (cine, literatura y música) como estrategias automotivadoras.

\section{6. ¿Qué estrategias crees que deben utilizar los/las profesores/as para motivar a los/as alumnos/as?}

Para contestar a esta pregunta, al ser las respuestas parecidas, las agrupamos por similitudes:

1er. Grupo (Lola, K Tatal y Jesús): Afirman que actividades lúdicas, dinámicas y de carácter cinético son estrategias que deben considerarse como motivadoras.

$2^{\circ}$. Grupo (Furilo, Lola, Ana, Vampira, Laura Ferrari y Tata): los profesores deben utilizar más las películas y los vídeos como estrategia motivadora dentro o fuera de la clase.

3er. Grupo (Tata, Laura Ferrari y Ane): destacan como EA los debates, diálogos y conversaciones (estrategia afectiva social -Oxford -) propuestos por los profesores/as.

$4^{\circ}$. Grupo (Simba y Pilar): se deberían preparar actividades fuera del contexto del aula como tareas relacionadas con el cine y a la música (estrategia afectiva).

$5^{\circ}$. Grupo (Guria y Lola): reiteran que actividades con música y canciones deberían ser más utilizadas por los profesores.

$6^{\circ}$ Grupo (Ana y Mari): piensan que la amistad entre docente y estudiante es una estrategia que también debe ser considerada como motivadora, además de otras. ñol).

${ }^{6}$ Los informantes muchas veces emplean el término «música« como sinónimo de «canciones« (en espa- 
$7^{\circ}$. Grupo (Machado y Edu): el estudio de la cultura y de las costumbres hispánicas deben ser los grandes factores motivadores para el alumnado.

$8^{\circ}$. Grupo (Juan y Lola): opinan que todas las estrategias usadas en el Centro de Estudios son muy buenas.

Estas respuestas demuestran que los encuestados clasifican las estrategias como «actividades» empleadas por los/las docentes. Es decir, aún sin advertir la complejidad para definir el concepto de estrategia, lo relacionaron conscientemente con «actividades». Por otro lado, cabe destacar la respuesta particular de Mari que ha resaltado el sentido del humor del profesor, un recurso apuntado por Rebecca Oxford (1990) también para reducir la ansiedad y la de Margi Simpson, que insinúa que la automotivación es la clave propulsora para el aprendizaje al creer que quien sólo estudia su propia lengua, no tiene posibilidades de progreso en la vida. Es decir, la estrategia sería el estudio del idioma en sí y lo que puede proporcionar su dominio (motivación extrínseca). En suma, películas, vídeos, juegos y dinámicas fueron apuntados como los preferidos entre este grupo de estudiantes como «actividades» más motivadoras.

\section{Además de las estrategias de aprendizaje que has marcado en el cuestionario, ¿utilizas otras?}

Esta pregunta se refería a otras estrategias particulares utilizadas por los/las estudiantes que no fueron previstas por el/la docente. La mayoría ha dicho que «no», que todas las EA que utilizan están en el cuestionario. Estos resultados demuestran que no hay mucha creatividad en el autoaprendizaje de los/las estudiantes o que tienen baja autonomía.

Para muchos, la «lectura» es una estrategia para ampliar el vocabulario, como han dicho: Ana (leer diccionarios, correos electrónicos, páginas web y otras curiosidades); Edu (leer cosas diversas); Lola y Margi Simpson (leer libros); Pilar (libros y revistas); y Machado (hacer investigaciones). Lola destaca el hecho de plantearse una meta, un objetivo, como en su caso era el de viajar a Buenos Aires, conocer otras culturas, etc. (motivación extrínseca «conseguir una recompensa»): su estrategia es la automotivación.

$\mathrm{Al}$ entrevistar a la profesora y preguntarle si trabaja alguna EA con su alumnado o piensa que cada uno debe desarrollar la suya, ella contesta que lo hace con frecuencia: que desarrolla estrategias con sus estudiantes que le hubieran servido a ella en el proceso de su aprendizaje y que repite muchas veces lo que sus profesores hacían. Menciona el hecho de elaborar carteles con textos y colgarlos en la pared, colocar la conjugación verbal en el espejo, etc.

La docente afirma que se debe caminar por la vía de la motivación de los/las estudiantes; es decir: «si les gusta la música, pues esto: a utilizarla en las clases». Esta postura supuestamente adoptada por la profesora tampoco es muy común entre muchos docentes, pues según afirman O'Malley et. al. (1985:21) en un estudio empírico con estudiantes y profesores de ESL (inglés/LE) en tres institutos de secundaria en EE. UU.: «teachers were generally unaware of students' strategies and rarely introduced strategies while teaching». Por otro lado, se observa que la docente utiliza mucho el sentido de humor en sus clases, quizá implícitamente («estrategia inconsciente»). Igualmente destaca que los/las aprendices disponen en casa de recursos como Internet y que a través de la red se puede escuchar la radio y leer periódicos en la lengua meta -lo que también podría motivarles -. En definitiva, esta profesora está 
convencida de que lo que más podría incentivar al alumnado sería enseñarles a estudiar; es decir, desarrollar la «competencia de aprendizaje» mediante el uso de estrategias metacognitivas: «esto es educar y este debe ser el papel del profesor-educador», señala.

\subsection{Resultados observables de las clases}

En las clases observadas se podrían obtener otras muchas conclusiones; no obstante, con relación al uso de estrategias vamos a desglosar unas cuantas:

- El uso del humor: Los relatos divertidos y anécdotas contadas por la docente constituyen, en sí mismas, estrategias, pues, a través de ellos, la docente conseguía reducir los niveles de ansiedad y establecía un ambiente más relajado. (Oxford, op. cit.)

- La negociación: La disponibilidad de la docente para negociar fechas de los seminarios demuestra que está atenta al ritmo individual de sus aprendices.

- Visionado de película: La profesora la secuencia en capítulos, entrenando la memoria y la expresión oral al tener que relatar lo visto en la clase anterior por lo que logra una mayor motivación (estrategia de memoria y cognitiva).

- Palabras de ánimo y de refuerzo: La docente aumenta la autoestima de su grupo transmitiéndole seguridad (estrategia de autoanimación -Oxford, op. cit.-).

- Digresión: Cuando la corrección de ejercicios se tornaba aburrida, la profesora utilizaba este recurso para volver a captar la atención del alumnado contando una historia divertida o algún hecho real relacionado con el tema.

- Utilización de la narrativa: La profesora les cuenta un relato amedrentador en lugar de corregirles explícitamente.

- Biorritmo: Una alumna (Lola) ha relatado que su estrategia particular era estudiar de madrugada pues le cundía más que durante el día -este era su biorritmo con relación a su estilo cognitivo-. (Oxford op. cit.) lo avala cuando escribe sobre las diferencias biológicas.

Ahora bien, a pesar de que «el uso del humor», «la negociación», «las palabras de ánimo y de refuerzo», «la digresión» y «el uso de la narrativa» se puedan clasificar, a modo de inferencia en este estudio, como «estrategias» utilizadas por la profesora para facilitar o mediatizar el proceso de enseñanza/aprendizaje, también ella misma sugiere a los estudiantes, en una de las clases observadas, unas cuantas «estrategias particulares» (estrategias del aprendiz) como hemos visto en el apartado anterior.

\section{Conclusiones}

Todas estas conclusiones, deducciones e inferencias extraídas de los cuestionarios y de las entrevistas nos ayudan a entender mejor el proceso de aprendizaje del español como lengua extranjera para lusoparlantes brasileños y, por lo tanto, facilitarían incluso la elección de EA más apropiadas, más motivadoras, además de materiales y de los procedimientos didácticos para favorecerlas aumentando su potencial de autonomía.

Las estrategias afectivas comprenden sentimientos, actitudes y motivaciones. Una vez que los aprendices están en contacto con esos factores afectivos, ellos pueden ejercer un mejor 
control sobre ellos, percibiendo sus emociones, sean positivas o negativas y evitando o controlando sus aspectos negativos.

Entre las estrategias de automotivación más comunes entre los aprendices encuestados destacamos el hecho de «hacer lecturas», «escuchar música» y «ver películas en español». A pesar de que Oxford no incluye estas «estrategias de automotivación» como el hecho de ver películas dentro o fuera de la clase, los alumnos encuestados sí que resaltan las artes (cine, literatura y música) como muy motivadoras. Es importante subrayar este tipo de estrategias una vez que los aprendices no están en un contexto de inmersión. Dicho de esta manera, el «aprendizaje» como proceso (opuesto a «adquisición») según la teoría de Krashen es la única vía posible, la cual, para nosotros, carecería de más recursos o estrategias motivadoras, que es precisamente lo que buscamos lograr con este estudio.

En muchas de las respuestas de los/as alumnos/as en las entrevistas, se relacionaron los términos «actividades» o «recursos» con el concepto de EA -es decir, para ellos son mecanismos más bien conscientes -

El uso del humor fue un recurso muy explotado por la profesora ${ }^{7}$ durante las clases observadas, lo que creó un ambiente de confianza y afectivamente propicio al aprendizaje, y sirvió también como estrategia de automotivación al obtener el feedback inmediato de sus alumnos cuando le sonreían o se reían.

En relación a los estilos de aprendizaje se advierte que hay diferencias individuales entre el estudiantado encuestado, pues se motivan con EA diferentes. No obstante, destacan sobre todo el estilo visual (películas, lecturas) y auditivo (música y canciones), pero también quizás el cinestético (actividades lúdicas, dinámicas, debates, etc.). Así es que por las diferentes EA específicas apuntadas se puede deducir también cuáles son sus preferencias y estilos, motivaciones y personalidades.

En definitiva, inferimos, por todo ello, que la motivación como componente afectivo parece tener un papel relevante para este grupo de estudiantes brasileños/as por el hecho de no encontrarse en un contexto de inmersión -por no ser el español su lengua oficial -. Apuntan algunas estrategias motivadoras como las relacionadas con el visionado de películas, la lectura de libros y las canciones, que fueron las más destacadas. También estamos de acuerdo con la profesora, quien en la entrevista sugiere que lo ideal es que cada estudiante desarrolle sus propias EA - «estrategias del aprendiz»- algo que brilla por su ausencia en el grupo en cuestión. Por todo ello, concluimos que los/las alumnos/as informantes no presentan ninguna EA creativa u original que no sean las ya conocidas o investigadas, lo que demuestra que quizá no sepan realmente como «automotivarse» o tengan bajos niveles de autonomía o de «competencia de aprendizaje».

7 Es cierto de que no se pueden confundir «estrategias» como «técnicas de trabajo» con la «acción mental» que interviene en los procesos de aprendizaje (EA), en este caso manejada por el aprendiz. En relación al uso del humor por parte de la docente, este se clasificaría como un «técnica de trabajo» (supuestamente de manera consciente) con un objetivo alcanzable: establecer un ambiente de confianza y afectivamente seguro entre todos. No obstante, Oxford (1990), clasifica «el uso del humor« como una estrategia afectiva que funciona para «reducir la ansiedad» regulando así las emociones: «Language learners, too, can benefit from laughter's anxiety-reducing powers. (...) Laughter is not just the result of teacher-centred joke-telling or Rassias-type dramatic; it can be stimulated by many kinds of classroom activities, such as role-plays, games, and active exercises in which learners are allowed to play as they learn.« (p. 164-165). 


\section{Bibliografía}

Alonso Tapia, J. y Cartula Fita, E. (2003). A motivação em sala de aula: o que é como se faz. São Paulo: Loyola.

Arnold, J. y Brown, D. (2000). «Mapa del Terreno». En J. Arnold, La dimensión afectiva en el aprendizaje de idiomas. Madrid: Cambridge.

Bernaus, M, Wilson, A. y Gardner, R. (2009). «Teachers' motivation, classroom strategy use, students' motivation and second language achievement». Porta Linguarum, 12: 25-36.

Díez, M. et al. (2004). Debate en torno a las estrategias de aprendizaje. Frankfurt: Peter Lang GMBH.

Ehrman et al. (2003). «A brief overview of individual differences in second language learning», System, 31: 313-330.

Ellis, R. (1985). Understanding Second language Acquisition. Oxford: OUP.

Fernández López, M. S. (1996). Estrategias de aprendizaje de la lengua extranjera. Madrid: Universidad Antonio de Nebrija.Fernández López, M. S. (2005). «Estrategias de aprendizaje» en Vademécum para la formación de profesores - Enseñar español como segunda lengua (L2) / lengua extranjera (LE). Madrid: SGEL.

Fernández López, P. (2006). «Entrenamiento Estratégico de la Comprensión Lectora como Lengua Extranjera. Las Estrategias Textual y Discursiva». Porta Linguarum, 6: 7-22.

Fonseca Mora, $\mathrm{M}^{\mathrm{a}}$ C. (2005). «Individual Characteristics of secondary school students». En N. McLaren, D. Madrid y A. Bueno (eds.) TEFL in Secondary Education. Granada: Editorial Universidad de Granada.

Franco Naranjo, P. (2004). «El uso de las estrategias de aprendizaje del inglés como lengua materna: aplicación de cuestionario como fase previa a la enseñanza de estrategias en el aula». Porta Linguarum 2: 57-67.

Gallardo, I. S. (1999). Lingüística aplicada a la enseñanza-aprendizaje del español como lengua extranjera. Madrid: Arcolibros.

Gardner, R. C. (2007). «Motivation and Second Language Acquisition». Porta Linguarum, 8: 9-20.

Gardner R. C. y Lambert W. E. (1972). Attitudes and Motivation in Second Language Learning. Rowley, Massachusetts: Newbury House.

Giovannini, A. et al. (2000). Profesor en acción, 1 - El proceso de Aprendizaje. Madrid: Edelsa.

Hill, S. J. (2004). «La falta de un consenso en la definición de una estrategia de aprendizaje». En Díez, M. et al. Debate en torno a las estrategias de aprendizaje. Frankfurt: Peter Lang GMBH.

Küster, L. (2004). «Recientes teorías motivacionales relevantes para la investigación sobre las estrategia de aprendizaje». En Díez, M. et al. Debate en torno a las estrategias de aprendizaje. Frankfurt: Peter Lang GMBH.

Madrid, D. (2000). «Learning Strategies». En Teaching English as a Foreign Language. Barcelona: The Australian Institute.

Marefat, F. y Barbari, F. (2009). «The Relationship between Out-of-Class language Learning Strategy Use and Reading Comprehension Ability». Porta Linguarum, 12: 91-106.

Martínez Lirola, M. (2004). «¿Qué relación guarda la motivación con los problemas de disciplina durante la adolescencia en los programas de educación bilingüe en EEUU?». Porta Linguarum 3: 21-34.

Manchón, R. M. (1993). La evolución del componente estratégico del aprendizaje de lenguas en Didáctica de ELE. Madrid: Col. Expolingua. 
Manchón, R. M. y Bruton, A. (1993). Serie sobre estrategias de aprendizaje y uso del lenguaje. Sevilla: CEP de Alcalá.

McLaren, N y Madrid, D. (eds.) (1996). A Handbook for TEFL. Alicante: Marfil.

Mohseni-Far, M. (2008). «A Cognitively-oriented Encapsulation of Strategies Utilized for Lexical Development: In search of a flexible and highly interactive curriculum». Porta Linguarum, 9: $35-52$.

Morales, M. y Smith, D. (2008). «Spanish Learning Strategies of Some Good Language Learners». Porta Linguarum, 9: 167-177.

O’Malley, J. M., Chamot, A. U., Stewner-Manzanares, G., Kupper, L. y Russo, R. P. (1985a). «Learning strategies used by beginning and intermediate ESL students». Language Learning, $35,1,21-46$.

O’Malley, J. M., Chamot, A. U. y Kupper, L. (1989). «Listening comprehension strategies in second language acquisition». Applied Linguistics 110/4:418-437.

O’Malley, J. M. y Chamot, A. U (1990). Learning Strategies in Second Language Acquisition. Cambridge: Cambridge University Press.

Oxford, R. L. (1990). Language Learning Strategies. What every teacher should Know. Heinle \& Heinle Publishers: Boston.

Padial Campón, R. y Tapia Carrillo, E. (2007). «The Motivation to use oral language in the ESL classroom in Secondary Education». Porta Linguarum 7: 135-151.

Sagnier, C. M. (2005). «L'apport des recherches sur les stratégies dans l'apprentissage d'une LE: vers de nouveaux modèles didactiques?» Porta Linguarum, 4: 77-85.

Savaiva, E. y Bernaus, M. (2008). «Motivación y Actitudes para el Aprendizaje de Lenguas de Dos Colectivos de Estudiantes Universitarios: Futuros Maestros de Lenguas Extranjeras y Futuros Enfermeros y Fisioterapeutas» Porta Linguarum, 10: 163-184.

Williams, M. y Burden, R. L. (1999). Psicología para profesores de idiomas. [(traducción: Alejandro Valero)]. Cambridge: Cambridge University Press. 